\title{
Les échos du passé. Enquête sur les débuts du Palika à Koné (Nouvelle-Calédonie, années 1970)
}

\section{Benoît Trépied}

\section{OpenEdition}

1 Journals

\section{Édition électronique}

URL : http://journals.openedition.org/jso/7868

DOI : $10.4000 /$ jso.7868

ISSN : 1760-7256

\section{Éditeur}

Société des océanistes

\section{Édition imprimée}

Date de publication : 15 décembre 2017

Pagination : 239-252

ISSN : 0300-953x

\section{Référence électronique}

Benoît Trépied, «Les échos du passé. Enquête sur les débuts du Palika à Koné (Nouvelle-Calédonie, années 1970) ", Journal de la Société des Océanistes [En ligne], 144-145 | 2017, mis en ligne le 15 décembre 2019, consulté le 15 mars 2021. URL : http://journals.openedition.org/jso/7868 ; DOI : https://doi.org/10.4000/jso.7868

\section{cc) (†) $\ominus$}

Journal de la société des océanistes est mis à disposition selon les termes de la Licence Creative Commons Attribution - Pas d'Utilisation Commerciale - Pas de Modification 4.0 International. 


\section{Les échos du passé. Enquête sur les débuts du Palika à Koné (Nouvelle-Calédonie, années 1970)}

par

Benoît TRÉPIED*

\section{RÉSUMÉ}

Dans les années 1970, la revendication d'indépendance kanak en Nouvelle-Calédonie a été portée par de nombreuses organisations partisanes, dont les principales étaient l'Union calédonienne et le Parti de libération kanak (Palika). En prenant appui sur une enquête ethnographique menée dans la commune rurale de Koné, cet article examine les logiques politiques et sociales de la différenciation entre ces deux partis. Au-delà des contextes historiques et des référents idéologiques et stratégiques distincts, l'implantation pratique du Palika à Koné reposait sur des clivages générationnels et territoriaux spécifiques, construits selon des modes d'historicité différents, qui révélaient des représentations kanak particulières du politique "sur le temps long", englobant et dépassant la seule problématique coloniale.

MoTS-CLÉs : nationalisme kanak, partis politiques, colonialisme, micro-histoire, Nouvelle-Calédonie

\begin{abstract}
In the 1970s, the Kanak claim for independence in New Caledonia was held by many political parties, including two major ones, the Union calédonienne and the Parti de libération kanak (Palika). Through ethnographic description of the rural municipality of Koné, this article stresses the political and social logics undermining the distinction between both parties. Apart from differences of contexts, strategies and ideologies, the practical implementation of the Palika in Kone relied on specific generational and territorial cleavages, elaborated through different modes of historicity, that revealed Kanak representations of politics "on the long run", including and going beyond the colonial issue.
\end{abstract}

KeYwords: Kanak nationalism, political parties, colonialism, micro-history, New Caledonia
Depuis son émergence dans les années 1970, la revendication d'indépendance kanak $^{1}$ a été portée en Nouvelle-Calédonie par une bonne douzaine d'organisations partisanes différentes: Foulards rouges, Groupe 1878, Parti de libération kanak (Palika), Union multiraciale, Union calédonienne (UC), Union progressiste mélanésienne, Front uni de libération kanak, Parti socialiste calédonien, Union syndicale des travailleurs kanak et exploités,
Libération kanak socialiste et, plus récemment, Rassemblement démocratique océanien, Front de coordination des comités indépendantistes, Parti travailliste, etc. (Leblic, 2003: 318-319; Chappell, 2013: 213-222). Au sein de cette mouvance nationaliste composite, chacun des partis a conservé son autonomie idéologique et organisationnelle, tout en s'investissant - pour la majorité d'entre eux - dans la construction

1. En 1774, James Cook a utilisé le mot d'origine polynésienne "Kanak» (signifiant homme) pour désigner les habitants de l'île baptisée par lui " Nouvelle-Calédonie ». Après la prise de possession française en 1853, ce terme a acquis une connotation péjorative sous la graphie francisée "canaque ". Lui étaient publiquement préférées les appellations " indigène ", puis " autochtone " ou " Mélanésien " à partir des années 1950. Dans les années 1970, les indépendantistes ont renversé le stigmate en faisant du mot « Kanak », restauré dans sa graphie initiale et invariable en genre et en nombre, un symbole de fierté culturelle et politique.

* Chargé de recherche, Institut de recherche interdisciplinaire sur les enjeux sociaux (IRIS, UMR 8156, CNRS-EHESs-InsermUniversité Paris 13), benoit.trepied@ehess.fr. 
de grandes coalitions, d'abord le Front indépendantiste (FI) en 1979, puis le Front de libération nationale kanak et socialiste (FLNKS) à partir de 1984. Ces dynamiques croisées de rapprochement et d'éloignement partisan invitent à s'interroger sur la structuration fluctuante $\mathrm{du}$ mouvement indépendantiste kanak: comment penser les spécificités de chacune des organisations partisanes le composant? Je souhaite plus particulièrement analyser ici les ressorts politiques et sociaux de la distinction entre l'Union calédonienne (UC) et le Parti de libération kanak (Palika), qui constituent depuis une quarantaine d'années les deux principaux partis indépendantistes, à l'échelle de la NouvelleCalédonie entière comme dans la commune rurale de Koné, au nord-ouest de la Grande Terre, où j'ai mené pendant deux années (2002-2004) une enquête ethnographique consacrée à la vie politique locale (Trépied, $2010: 14-17)^{2}$.

Ces deux organisations militantes ne sont pas nées au même moment et dans des conditions identiques. Créée quelques années seulement après la transformation statutaire de cette colonie de peuplement française du Pacifique en territoire d'outre-mer et l'extension de la citoyenneté aux ex-« sujets indigènes » en 1946, l'uc a occupé le devant de la scène politique pendant les années 1950 et 1960, à une époque où la revendication d'indépendance n'était pas formulée. Doté du slogan "deux couleurs, un seul peuple", le parti regroupait les suffrages d'une importante minorité des électeurs européens (descendants des colons) et de la grande majorité des électeurs mélanésiens, au sein d'une population calédonienne totale d'environ 65000 habitants en 1951, dont $52 \%$ d'autochtones et $32 \%$ d'Européens ${ }^{3}$. Le vote des citoyens mélanésiens était alors strictement encadré par deux grandes associations modérées créées au lendemain de la guerre par les Missions chrétiennes pour contrer la propagande communiste naissante : l'Union des indigènes calédoniens amis de la liberté dans l'ordre (UICALO), catholique, et l'Association des indigènes calédoniens et loyaltiens français (AICLF), protestante (Kurtovitch, 1997). Ces deux mouvements avaient fondé leur autorité sur le réseau des paroisses implantées dans toutes les "tribus" et sur l'influence locale des notables religieux indigènes (pasteurs, séminaristes, diacres, catéchistes) et des " chefs " administratifs ${ }^{4}$. Après avoir rapidement défait le mouvement communiste, puis soutenu la dynamique politique qui allait aboutir à la création de l'UC, l'UiCALO et l'AICLF reconvertirent électoralement les relations de pouvoir qu'elles exerçaient sur les habitants des tribus en les incitant à voter en faveur des candidats du parti. Le fort taux de participation et l'adhésion massive des électeurs kanak aux candidats de l'uc dans les années 1950 soulignaient l'efficacité de ces "médiations ethno-religieuses du vote" (Soriano, 2014 : 105).

À la fin des années 1960, les grandes transformations sociales et économiques qu'a connues la Nouvelle-Calédonie ont profondément modifié la situation politique. Lors du fameux «boom du nickel »(1968-1972), l'arrivée de nombreux migrants originaires de métropole et des autres territoires français du Pacifique a placé pour la première fois les Kanak en position de minorité démographique : en 1976, ils ne représentaient plus que $42 \%$ des 133000 habitants 5 . Simultanément, l'accroissement du niveau de vie et la modernisation des infrastructures n’ont bénéficié que marginalement aux Kanak, accroissant d'autant les frustrations sociales (Freyss, 1995: 293-310). Par ailleurs, alors que le premier bachelier mélanésien n'était recensé qu'en 1962, les premiers étudiants kanak formés dans les universités métropolitaines sont retournés sur le territoire au tournant des années 1970 avec un nouveau regard sur la société calédonienne. Influencés par les idéologies marxistes et tiersmondistes alors en vogue, certains d'entre eux ont lancé de nouveaux mouvements contestataires: créés en 1969 et 1974 et recrutant respectivement des militants kanak des îles Loyauté (Maré, Tiga, Lifou, Ouvéa) et de la Grande Terre (île principale), les « Foulards rouges " et le " Groupe 1878 » ont d'abord mobilisé de nouvelles catégories dans le discours politique - dénonçant le « colonialisme » subi par le "peuple kanak» - avant d'émettre publiquement la première revendication d'indé-

2. La circonscription communale de Koné englobe un "village » européen et neuf «tribus " (hameaux kanak) : Atéou, Baco, Bopope, Koniambo, Néami, Netchaot, Noelly, Tiaoué et Poindah (où une famille kanak m’a accueilli pendant ces deux années). Mon enquête repose sur de nombreux entretiens et observations ethnographiques réalisés sur l'ensemble du territoire communal, complétés par un travail sur archives. Les patronymes et toponymes apparaissent ici sous leur graphie francisée, tels qu’ils étaient couramment utilisés et prononcés au moment de l'enquête.

3. Population de la Nouvelle-Calédonie au 10 octobre 1951, Archives de la Nouvelle-Calédonie 37W351. Ce document décomposait la population calédonienne en quatre catégories : " Européens », " Autochtones », " Indochinois » (6 \%) et "Indonésiens" $(10 \%)$. La Nouvelle-Calédonie est toujours aujourd'hui le seul territoire de la République française autorisé à produire des statistiques « ethniques».

4. Selon les catégories de l'administration coloniale, une " tribu » était une entité socio-politique située sur une « réserve indigène » et représentée par un « chef » administratif nommé et rémunéré par le gouverneur. Dans le langage courant, une tribu désigne désormais un village kanak.

5. Population de la Nouvelle-Calédonie selon la communauté d'appartenance aux différents recensements, document en ligne (consulté le 23 décembre 2016) : www.isee.nc/population/recensement/communautes. Sur l'évolution de la population kanak dans la population globale, voir aussi Leblic, 1993 : 30-36. 
pendance en janvier 1975, puis de fusionner l'année suivante pour former le Palika (Chappell, $2003)^{6}$. D'autres intellectuels, à l'instar de JeanMarie Tjibaou (1936-1989), se sont lancés dans un travail de revalorisation culturelle, culminant lors du festival "Mélanésia 2000 " en septembre 1975, avant de s'engager en politique (JSO, 1995; Tjibaou, 1996). Sous leur impulsion, l'uc vota à son tour une motion en faveur de l'indépendance kanak en 1977, suscitant le départ de la plupart des militants européens, tandis que Jean-Marie Tjibaou et d'autres nouveaux leaders (Yeiwéné Yeiwéné, Éloi Machoro, Pierre Declercq, François Burk) prenaient la direction du parti ${ }^{7}$.

Cette genèse politique et intellectuelle plurielle du nationalisme kanak permet de saisir en partie les logiques de différenciation entre l'uc et le Palika dans les années 1970. Comprenant en leur sein de nombreux dissidents kanak de l'uc mais aucun Européen (après mars 1970), les Foulards rouges et le Groupe 1878 se distinguaient de l'ancien parti majoritaire en proposant une grille d'analyse marxiste qui privilégiait l'appréhension de la situation coloniale en termes de classes sociales et de matérialisme dialectique; ils critiquaient radicalement le jeu électoral et institutionnel et organisaient des actions "de terrain " (manifestations, désobéissance civile, etc.), en particulier - pour le Groupe 1878 des opérations de revendications foncières sur les propriétés des colons (Guiart, 2001 ; Leblic, 1993 : 194-209; Demmer, 2016 : 21-28). De son côté, condamnant ces dissensions partisanes, l'uc luttait en faveur de l'indépendance en se concentrant largement (mais pas uniquement) sur les questions liées à l'identité kanak et en assumant plus volontiers sa participation au travail politique mené dans les institutions représentatives; elle a par ailleurs toujours conservé en son sein quelques militants et dirigeants européens. Dans les représentations communes comme dans la presse calédonienne et métropolitaine, ces divergences idéologiques et stratégiques ont contribué à construire l'image classique d'un mouvement indépendantiste clivé entre un parti radical marxiste et un parti modéré d'origine religieuse. Quand on y regarde de plus près, ces antagonismes apparaissent pourtant relatifs : les préoccupations identitaires et culturelles du Palika (Chappell, 2013 : 156159; Néaoutyine, 2006: 129-130) faisaient à cet égard écho aux revendications sociales et économiques de l'uc (Tjibaou, 1996 : 143-149), ce qui permit d'ailleurs l'élaboration du projet commun "d'indépendance kanak socialiste (IKS) » au sein du FI et du FLNKS - même si l'UC et le Palika n'y mettaient pas au départ exactement la même chose - puis l'investissement similaire des deux partis dans les politiques de développement depuis les années 1990 (Leblic, 1993 ; Demmer, 2016). Mais surtout, cette approche "idéelle » de la vie politique calédonienne ne rend pas compte des logiques pratiques de la différenciation partisane : elle fait l'impasse sur une histoire sociale de la distinction entre l'uc et le Palika susceptible de révéler les mécanismes non proprement politiques en jeu dans le processus de fixation des appartenances partisanes.

A contrario, je cherche ici à comprendre ce qui différenciait socialement les deux principales organisations indépendantistes. Le politiste Éric Soriano souligne à ce sujet que :

"l'indistinction sociologique des électorats de chaque organisation mélanésienne ne fournit pas d'assises à des distinctions idéologiques. [...] À quelque niveau que l'on se situe (communal, "tribal" ou même communautaire), aucun indice ne permet d'affirmer que l'appartenance à telle ou telle catégorie socialement objectivable prédispose à un vote en faveur d'une organisation ou d'un type de discours. " (Soriano, 2001: 122-123)

Il me semble pourtant possible, en resserrant la focale à l'échelle de la commune de Koné, de dépasser ce constat sur l'indistinction sociologique de la différenciation partisane au sein de la mouvance indépendantiste. Le recours à la micro-analyse permet en effet de révéler un certain nombre de clivages sociaux, certes difficilement repérables à l'échelon territorial car indépendants des grandes variables socioprofessionnelles classiques, mais localement pertinents dans le cadre du processus de cristallisation des diverses appartenances partisanes. En étudiant les modalités de la différenciation uc/Palika dans la région de Koné, cet article a donc pour ambition de saisir la façon dont les logiques politiques générales au fondement de cette distinction partisane se sont articulées à d'autres logiques sociales locales.

Ces "jeux d'échelles" (Revel, 1996) ne se déploient pas seulement dans l'espace mais également dans le temps. L’analyse localisée des modes d'affiliation partisane met ainsi au jour des configurations politiques et sociales qui ne correspondaient pas au seul cadre historique

6. Les premiers graffitis pour l'indépendance ont été peints sur les murs de Nouméa en juillet 1969 par un petit groupe d'étudiants européens radicaux alliés aux étudiants kanak. Dès mars 1970, ces activistes européens et kanak décidèrent conjointement et stratégiquement de créer des structures militantes séparées racialement (Chappell, 2013: 87, 101). Le nom du "Groupe 1878 " renvoyait à une grande révolte kanak qui secoua la colonie cette année-là.

7. Dès le 25 juin 1975 à la tribu de La Conception, soit avant même l'émergence de cette nouvelle génération de dirigeants, les « vieux " élus kanak de l'uc avaient signé en leur nom propre - mais pas au nom de leur parti - une déclaration commune en faveur de l'indépendance kanak, aux côtés de l'Union multiraciale et des groupes à l'origine du Palika (Leblic, $1993: 60-61)$. 
de la confrontation entre l'uc et le Palika mais renvoyaient aussi à d'autres rapports au passé. Certaines logiques d'appartenance politique s'inscrivaient dans la temporalité de la "paix coloniale» (1920-1970) où furent enracinées, à l'intérieur des réserves indigènes, les nouvelles institutions de contrôle social en tribu (églises et chefferies administratives) qui créèrent les conditions du succès politique de l'uc après 1946 (Trépied, 2010 : 51-81). D'autres convoquaient les mémoires familiales et locales des confrontations antérieures face aux Européens à la fin $\mathrm{du} \mathrm{XIX}^{\mathrm{e}}$ et au début du $\mathrm{Xx}^{\mathrm{e}}$ siècles : spoliations foncières massives, affrontements militaires (notamment lors des guerres de 1878 et 1917), déplacements de population, puis enfermement dans les réserves et instauration du régime de l'Indigénat (Saussol, 1979; Dauphiné, 1989 ; Merle, 2004 ; Muckle, 2012). Enfin la distribution des orientations partisanes à Koné renvoyait également à des dynamiques politiques kanak propres qui dépassaient le seul « moment colonial». Celles-ci révélaient :

" des manières locales spécifiques de penser le rapport à la terre, aux ancêtres, à la richesse et au pouvoir [... qui] ont perduré par-delà les césures de "temps court" induites par l'implantation commerciale, missionnaire, militaire et administrative des Européens. " (Bertrand, 2006 : 21)

L'analyse des conditions de la réception de la propagande du Groupe 1878 puis du Palika renvoie donc à des configurations sociales originales, liées à des modes d'historicité particuliers du politique, qui conditionnèrent le lancement du parti dans la région. Les modalités de l'implantation du Palika à l'intérieur de la commune de Koné relevaient plus précisément du croisement de deux logiques complémentaires - la première générationnelle, la seconde territoriale - engageant des rapports différents au passé, que nous allons successivement examiner.

\section{« Jeunes » contre « vieux »?}

\section{Mobiliser "les jeunes »}

Les récits que j'ai recueillis au début des années 2000 sur la genèse des organisations partisanes à l'origine du Palika décrivent systématiquement ces mouvements comme des groupes politiques de « jeunes ${ }^{8}$. Né en 1945, Elie Poigoune a été l'un des premiers étudiants kanak à Montpellier dans les années 1960, avant d'être recruté comme professeur de mathématiques au lycée Lapérouse de Nouméa au début de la décennie suivante. En 1974, aux côtés de quelques autres militants originaires de la Grande Terre (Déwé Gorodé, Gabriel Moentéapo, Edmond Koataiba, Madeleine et Bernadette Ayawa, etc.), il fut l'artisan principal de la création du Groupe 1878 (Chappell, 2013: 131-133)9. Dans un entretien qu'il m'a accordé en 2003, il insistait rétrospectivement sur l'appartenance des premiers militants à cette catégorie sociale des « jeunes », fréquemment mobilisée dans les discours kanak contemporains (Salaün, 2009) :

«Au départ, c’est dans les années 70, 72, 73, 74. On était des étudiants, on revenait, on avait vécu un peu Mai 68 en France. [...] On a décidé, avec des jeunes de la Grande Terre, de faire un peu le même travail qu’a fait Nidoïsh Naisseline [fondateur des Foulards rouges] dans les îles Loyauté. [...] On a dit qu'il faut regrouper les jeunes, comme on faisait partie des milieux jeunes. Les milieux à l'époque, c'est assez fermé, il y a des coutumiers, il y a des religieux... Donc sur la Grande Terre on a regroupé des jeunes, à Nouméa. » (Élie Poigoune, entretien du 15 décembre 2003, Nouméa $)^{10}$

En pratique, ces premiers "milieux jeunes" investis par le Groupe 1878 renvoyaient essentiellement à trois sous-groupes kanak: les habitants de Nouméa, les ouvriers du chalandage séjournant temporairement au chef-lieu et les collégiens et lycéens scolarisés dans les principaux établissements du territoire. À l'époque, ces diverses scènes sociales - à la ville, au port et à l'école - constituaient pour la population kanak les principaux espaces de résidence et de circulation à l'extérieur des réserves. De ce fait, elles devinrent rapidement des foyers privilégiés de contestation politique où les nouveaux mouvements radicaux diffusèrent leur propagande. Citons les témoignages de deux des premiers militants du Groupe 1878 originaires de Koné, plus précisément de la tribu de Netchaot, respectivement âgés de 30 et 15 ans en 1974 :

"Nous, on va toujours au chalandage, ça fait que les discussions elles sont parties, commencer à discuter du parti, donner un peu des informations. [...] C'est là où on a eu des contacts, il y avait déjà les groupes, Foulards rouges, tout ça, pour nous, comme c'était à Nouméa. " (Léon Poudéwa, entretien du 22 mai 2004, tribu de Netchaot, Koné)

8. Christine Demmer (2016 : 79-99) fait un constat identique dans son enquête sur les militants Palika des années 1970-1980 à Canala (côte est).

9. Sur la création du Groupe 1878, on peut aussi consulter une longue interview de Gabriel Moentéapo (1945-2013) réalisée au cours des années 2000 et disponible en ligne (consulté le 23 janvier 2017) : http://archives.caledosphere. com/2014/05/24/page-dhistoire-temoignage-de-gaby-monteapo/.

10. Toutes les citations d'Élie Poigoune dans la suite du texte sont issues de cet entretien. 
"J'étais à l'école, au collège protestant de Do Néva, à Houaïlou [côte est], en 1968. Vers 1968-69, il y a un journal qui est passé, c'est le journal des Foulards rouges. [...] C'est un journal qui passait à Do Néva, c'est une école protestante, il y a beaucoup de Kanak qui sont dedans. Le journal, il passe entre nous. C'est là que j'ai commencé à lire les trucs, et puis ça a accroché, "personnalité kanak", "identité kanak”. [...] Ça a continué comme ça, il y a eu des discussions entre nous les lycéens. Après, vers 1974, je suis entré en seconde au lycée Blaise Pascal à Nouméa. C'est là qu'ils ont créé le Groupe 1878, c'était Élie Poigoune, Déwé Gorodé, Gabriel Moentéapo, tout ça là. Moi, j’avais un oncle qui était toujours avec eux. Il m'a amené dans le Groupe 1878. C'est là que j'ai commencé à militer. » (Joseph Goromido, entretien du 30 septembre 2002, village de Koné)

À ces premiers militants étudiant ou travaillant à l'extérieur des réserves se rajoutèrent ensuite d'autres jeunes vivant en tribu. Dès sa création, le Groupe 1878 eut en effet vocation à poursuivre et à adapter pour l'ensemble de la Grande Terre le travail de "conscientisation" politique mené aux îles Loyauté par les Foulards rouges depuis 1969. En 1974 et 1975, les fondateurs du mouvement organisèrent dans ce but de grandes tournées d'information dans l'ensemble des tribus de l'île principale, "avec le bâton de pèlerin » selon Élie Poigoune :

"On circulait, on partait deux semaines, on installait notre tente quelque part, et puis on allait faire la coutume à une tribu, demander qu'on fasse une réunion. [...] On était maximum une dizaine, hein. On prenait une voiture, une camionnette, et puis on allait. On commençait du côté de Bourail, parce que les Kanak c'est surtout à partir de là. On faisait tribu par tribu. [...] Moi, j’enseignais déjà ici [au lycée Lapérouse de Nouméa], donc on faisait ça pendant nos vacances. Notre travail, c'était surtout l'information. »

Pour avoir une idée plus précise des frontières du groupe "jeunes " évoqué dans ces souvenirs, j'ai comparé les dates de naissance des principaux leaders et des premiers militants du Palika originaires de la région de Koné que j'ai pu identifier au fil de mon enquête ethnographique, soit un corpus d'une douzaine d'individus. Ils appartenaient tous aux classes d'âge nées dans les années 1940 ou 1950 : au milieu des années 1970 , les plus jeunes militants avaient environ 15 ans et les plus âgés un peu plus de 30 ans. Ce groupe s'inscrivait donc dans un phénomène générationnel (Mannheim, 1990), puisque ses membres «ont partagé des expériences de nature similaire qui les ont conduits à s'accorder sur certains objectifs sociopolitiques " (Demmer, 2003 : 5). Les transformations du vécu kanak découlant du changement social et politique de 1946 - suppression du régime de l'Indigénat et accession à la citoyenneté - jouaient ici un rôle crucial dans la formation du groupe générationnel spécifique qui s'engagea trente ans plus tard dans la lutte d'indépendance (Demmer, 2016 : 73-76).

Cela étant dit, toute la jeunesse kanak du milieu des années 1970 n'était pas Palika : le virage nationaliste avait tout autant concerné les jeunes qui restèrent dans l'uc derrière JeanMarie Tjibaou. Au conseil municipal de Koné par exemple, entre 1983 et 2001, sur vingt-neuf élus indépendantistes nés entre 1940 et 1960, quinze étaient des militants de longue date de l'uc contre douze du Palika. Dans ce contexte, la spécificité des jeunes qui rejoignirent le Groupe 1878 résidait essentiellement dans la rupture politique que leur engagement partisan suscitait vis-à-vis de leurs aînés : l'adhésion à la mouvance Palika représentait un refus de suivre la ligne UC tracée par leurs parents. Si au niveau des états-majors, d'après Élie Poigoune, les jeunes fondateurs du Palika bénéficiaient du soutien discret mais réel de certains "vieux " dirigeants kanak de l'uc (Gabriel Païta, Eugène Ayawa, Similien Nahiet, Jean-Marie Chanene, etc.), en pratique et à l'échelle locale, la dynamique militante Palika signifiait de facto une contestation de la domination politique exercée par l'uc en tant qu'organisation partisane. C'était particulièrement le cas dans la commune de Koné, véritable " fief UC » où le parti occupait au tournant des années 1970 une position quasiment hégémonique parmi les Kanak de la région, et où, donc, tous les jeunes militants du Palika avaient des parents $\mathrm{UC}^{11}$.

\section{Rupture générationnelle et histoire coloniale}

Cette position de rupture politique des jeunes du Palika vis-à-vis de leurs aînés avait ceci de paradoxal qu'elle se référait à un discours sur le passé qui justifiait l'engagement militant contemporain à l'aune de l'histoire coloniale. En 1974, le Groupe 1878 mobilisait ainsi l'argument des spoliations foncières, plus de 70 ans après les faits: selon Élie Poigoune, " notre première revendication, dans ce groupe, c'était "rendez-nous nos terres", il fallait rendre les terres qui avaient été volées par les colons, les rendre aux Kanak». Dans le cadre de leur propagande comme à travers le nom de leur groupe ("1878», en référence à la grande insurrection kanak de cette année-là), les leaders étudiants mettaient un soin particulier à tisser des liens entre leur mouvement et les anciennes

11. L'Union multiraciale n'était quasiment pas implantée à Koné. Une partie non négligeable des habitants de la tribu de Poindah avait quitté l'uc dès le début des années 1960 à la suite d'une crise politico-religieuse (voir plus loin). Ils rejoignirent ensuite non pas le Palika mais, à l’opposé du spectre politique, le camp anti-indépendantiste (Trépied, 2010 : 165-194). 
mobilisations kanak contre les colons ou l'armée française, jetant un pont par-dessus cinquante ans de paix coloniale. Ce faisant, ils incitaient leurs militants à se réapproprier leurs histoires familiales antérieures à 1920 et à inscrire leur engagement dans cette perspective. Après avoir évoqué le souvenir de son grand-père insurgé lors de la guerre de 1917, l'un des premiers militants originaires de la tribu de Baco, interrogé en 2003, soulignait le rôle joué par les dirigeants du Palika dans ce processus de prise en charge politique de son héritage familial :

BT : «Est-ce que cet héritage familial joue un rôle dans ton engagement politique?"

Émile My: "Surtout moi, bien sûr. Moi, par rapport au parcours de mon grand-père, on a su avec nos leaders politiques qui sont Élie Poigoune, Paul Néaoutyine, on a su remonter l'histoire de chacun. Et c'est pour ça, nous, toute la famille depuis, nous sommes toujours restés Palika. » (Émile My, entretien du 15 avril 2003, tribu de Baco, Koné)

Nés autour de 1946, ces premiers militants du Palika n'avaient pas connu les effets immédiats des guerres et du refoulement foncier de la période 1860-1920. La mémoire des injustices coloniales, transmise selon des modalités particulières au fil des générations (Naepels, 1998 : 127-141), semblait en fait d'autant plus fortement mobilisée dans l'espace militant par ces jeunes que leur éloignement social et temporel vis-à-vis du monde kanak de l'époque coloniale était important. À l'inverse, les "vieux» des années 1970, nés peu de temps après la dernière grande vague de spoliations foncières (le " grand cantonnement" de 1899-1903 organisé par le gouverneur Feillet), avaient grandi dans un milieu social et familial profondément marqué par cette perte (Demmer, 2002 : 201-253). Pour eux, la soumission directement vécue au régime de l'Indigénat et les contrecoups immédiats du choc colonial qu'avaient subi leurs propres parents à la fin du $\mathrm{XIX}^{\mathrm{e}}$ siècle constituaient un socle commun d'expériences sociales, pour une large part traumatiques, à l'aune duquel les réformes en tribu mises en œuvre par l'UC après 1946 (notamment en termes d'infrastructures: conduites d'eau, routes, etc.) avaient constitué une amélioration marquante des conditions de vie (Trépied, 2010 : 51-76). Les changements concrets liés à la citoyenneté et au vote, dont ils avaient été témoins et acteurs, justifiaient dans les années 1970 la continuité et la force de leur attachement militant à l'uc.

Pour les jeunes du Palika en revanche, l'obligation sociale du respect dû aux vieux faisait en quelque sorte l'objet d'un saut générationnel : l'évocation de leurs aïeux du XIX ${ }^{e}$ siècle, spoliés de leurs terres et combattus par l'armée française, légitimait leur propre rupture partisane vis-à-vis de leurs ascendants encore en vie qui soutenaient l'uc. Ce faisant, ils dénonçaient dans un même mouvement non seulement l'époque des violences coloniales (1853-1917) puis celle d'une colonisation "pacifiée " non moins oppressante (1917-1946), mais aussi l'ère de la citoyenneté (à partir de 1946) et le réformisme modéré de l'Uc (années 1950-1960). Cette dernière était réduite à une simple entreprise de manipulation des électeurs mélanésiens, comme le résumait le journal des Foulards rouges en 1970 en dénonçant la mainmise des dirigeants européens de l'UC:

«Ce parti se sert de vous pour attirer la masse électorale canaque afin de toujours maintenir à la tête du pays une oligarchie d'exploiteurs à la solde du colonialisme. " (Réveil canaque 2, 30 septembre 1970)

Les groupes à l'origine du Palika condamnaient également l'immobilisme, voire la complicité des Eglises et des chefferies administratives vis-à-vis du phénomène colonial, comme en témoigne l'article "La coutume et la lutte» paru dans Colonisé, qui se terminait par :

«À bas les chefs fantoches! Vive la coutume au service du peuple !» (Colonisé 2, 21 septembre 1979, in Soriano, $2001: 377$ )

Au début des années 1970, ce type de discours représentait une menace pour l'ordre social stabilisé dans les réserves depuis le début du $\mathrm{XX}^{e}$ siècleautour des autorités indigènes religieuses et administratives, dont l'action après-guerre avait été déterminante pour la formation d'un électorat kanak pro-Uc. Les formes contestataires de mobilisation collective, proposées par les Foulards rouges et le Groupe 1878, entraînant fréquemment des affrontements avec la police à Nouméa, contribuaient également à briser la paix coloniale ininterrompue depuis les années 1920. Dans le cas de Koné (et probablement aussi ailleurs), cette stratégie de rupture eut pour effet de susciter de fortes tensions à l'intérieur des tribus entre les vieux de l'uc et les jeunes du Palika, les premiers stigmatisant les seconds, selon les témoignages recueillis sur place, comme des " révolutionnaires" ou des "bordéliques». Par conséquent, d'après mes interlocuteurs, jusqu'au tournant des années 1980, les réunions du Palika dans les tribus de Koné étaient généralement organisées en cachette des vieux. Ces tensions pouvaient se déployer jusqu'au cœur même des familles, à l'image du témoignage de Joseph Goromido, maire actuel Palika de Koné dont le père Antoine était un responsable local de l'uc :

«Pour moi, c'est ça, c'est la dispute tous les jours. Parce que nous là-haut à la maison, on se dispute tout le temps. Moi avec mon père. On se dispute toujours. [...] C'est conflictuel, c'est uc-Palika. Dans mon cas personnel, c'est ça, ça a pas été facile quoi. Seulement 
on a vécu ensemble, la famille, la coutume, tout ça, mais quand on aborde la politique, c'est la dispute quoi. C'est sûr, parce que nous on critique: "Vous à l'uc, vous êtes des petits bourgeois !" Moi avec le vieux [son père] ! Le vieux il dit: "Ouais mais révolutionnaires, vous allez faire quoi pour le pays, vous les révolutionnaires ! ?" "Ben nous, on veut construire le socialisme !" "Quand les poules auront des dents oui !" [nous rions]. C'était conflictuel quoi. » (Joseph Goromido, entretien du 30 septembre 2002, village de Koné)

Contrairement aux militants du Palika, les jeunes de l'uc se plaçaient eux dans la continuité politique de la génération de leurs parents: à leurs yeux, le respect dû aux vieux justifiait de rester dans le parti qu'ils avaient reçu en héritage, quitte à le transformer de l'intérieur en organisation nationaliste kanak. Ce discours de légitimation était en outre articulé au bilan des politiques publiques menées depuis les années 1950 par l'uc au profit des Kanak. Âgé de 21 ans en 1974 et originaire de la tribu de Tiaoué, Yvon Goromoedo mobilisait ces deux types d'argument pour expliquer sa fidélité à l'uc lors de notre entretien de juillet 2003 :

"Nous, toute la famille, les cousins, tout ça, on est UC, parce que nos vieux, ils sont trois, les trois frères, avec mon papa. Ils nous disent toujours : "vous voyez, l'uC, si aujourd'hui on a tout, c'est grâce à lui, c'est pas un autre. Alors nous on est UC, vous aussi. Restez dans l'uc". Ça fait que maintenant, on est toujours uc. Ça reste toujours. Dans toute la famille, même les générations d'après, ils vont rester, parce que nous on va transmettre le message: "aujourd'hui, t'es à l'école, tu mets des chaussures, mais avant nous on met pas de chaussures. Tout ça, le téléphone, tout ça, c'est l'uc." " (Yvon Goromoedo, entretien du 2 juillet 2003, village de Koné)

Les arguments avancés pour justifier l'adhésion à l'uc s'inscrivent ici dans un rapport particulier au passé qui n'est pas sans rappeler celui des partisans Palika. Dans les deux cas, l'engagement militant dans les années 1970 était d'abord appréhendé à la lumière de l'histoire coloniale telle qu'elle était déclinée dans les mémoires locales et familiales, notamment ici sous l'angle du sous-équipement chronique des tribus pendant la période coloniale. La seule différence - de taille - résidait dans le fait que les jeunes de l'uc ne brisaient pas le processus de transmission générationnelle des dispositions politiques reçues de leurs parents, inversement à ceux du Palika qui légitimaient leur opposition à l'uc en invoquant les destins de leurs aïeux des générations précédentes.

$\mathrm{Si}$ donc un décalage générationnel dans le rapport au passé et à la politique explique l'apparition d'un important conflit entre jeunes du Palika et vieux de l'uc, l'opposition entre les deux partis ne recouvrait pas exactement le clivage jeunes/vieux à l'échelle de la commune de Koné. Elle renvoyait également à une distribution différenciée des appartenances partisanes dans l'espace, dont il convient d'étudier maintenant les logiques sociopolitiques.

\section{Les chemins du Groupe 1878 à Koné}

Concernant les premières tournées du Groupe 1878 à Koné en 1974-1975, les témoignages que j'ai recueillis dans la région au début des années 2000 ne mentionnent que la présence et l'action du seul Élie Poigoune, fondateur du mouvement, comme s'il avait été l'unique militant à circuler dans la région à cette époque - alors même qu'il évoque des tournées concernant "maximum une dizaine " de personnes (cf. supra). L'éventuel rôle de médiation joué par d'autres militants originaires de Koné ou d'ailleurs (notamment les locuteurs natifs du paicî - principale langue kanak parlée à Koné - comme l'étaient les cofondateurs du Groupe 1878 Déwé Gorodé ou Gabriel Moentéapo, originaires de Ponérihouen sur la côte est), n’est en revanche jamais évoqué dans les entretiens, ni dans les très rares archives partisanes locales que j'ai pu consulter. À défaut de sources écrites, cette convergence des sources orales sur le rôle politique fondamental d'Élie Poigoune - lui-même locuteur du cèmuhî, langue parlée à Koné dans les deux tribus de Bopope et Netchaot - invite à analyser précisément les conditions dans lesquelles il tenta de lancer le Groupe 1878 à Koné.

En l'occurrence, la première de ces conditions, qui se révéla a posteriori la plus importante stratégiquement, concernait la possibilité ou l'impossibilité, pour Élie Poigoune, d'être reçu dans les tribus et d'y tenir des réunions d'information politique. On peut en effet observer que le Palika " prit » là où son fondateur fut autorisé à s'installer et à diffuser la propagande du parti. C'était le cas dans cinq des neuf tribus incluses dans la circonscription de Koné : Baco, Bopope $^{12}$, Néami, Netchaot et Noelly. Par contre, l'hostilité des habitants des quatre autres tribus de Koné (Tiaoué, Poindah, Koniambo et Atéou) contre le Palika se manifesta dès l'origine par le refus de laisser Élie Poigoune entrer et/ou organiser des réunions dans ces tribus. L'étude de l'implantation du Groupe 1878 à Koné passe donc ici par une analyse "au ras du sol» des logiques sociales et politiques mobilisées à l'occasion des tournées locales de l'organisation : pour quelles raisons Élie Poigoune était-il accepté

12. La tribu de Bopope est située à l'intérieur de la circonscription communale de Poindimié (côte est) mais rattachée administrativement à la commune de Koné (côté ouest). 


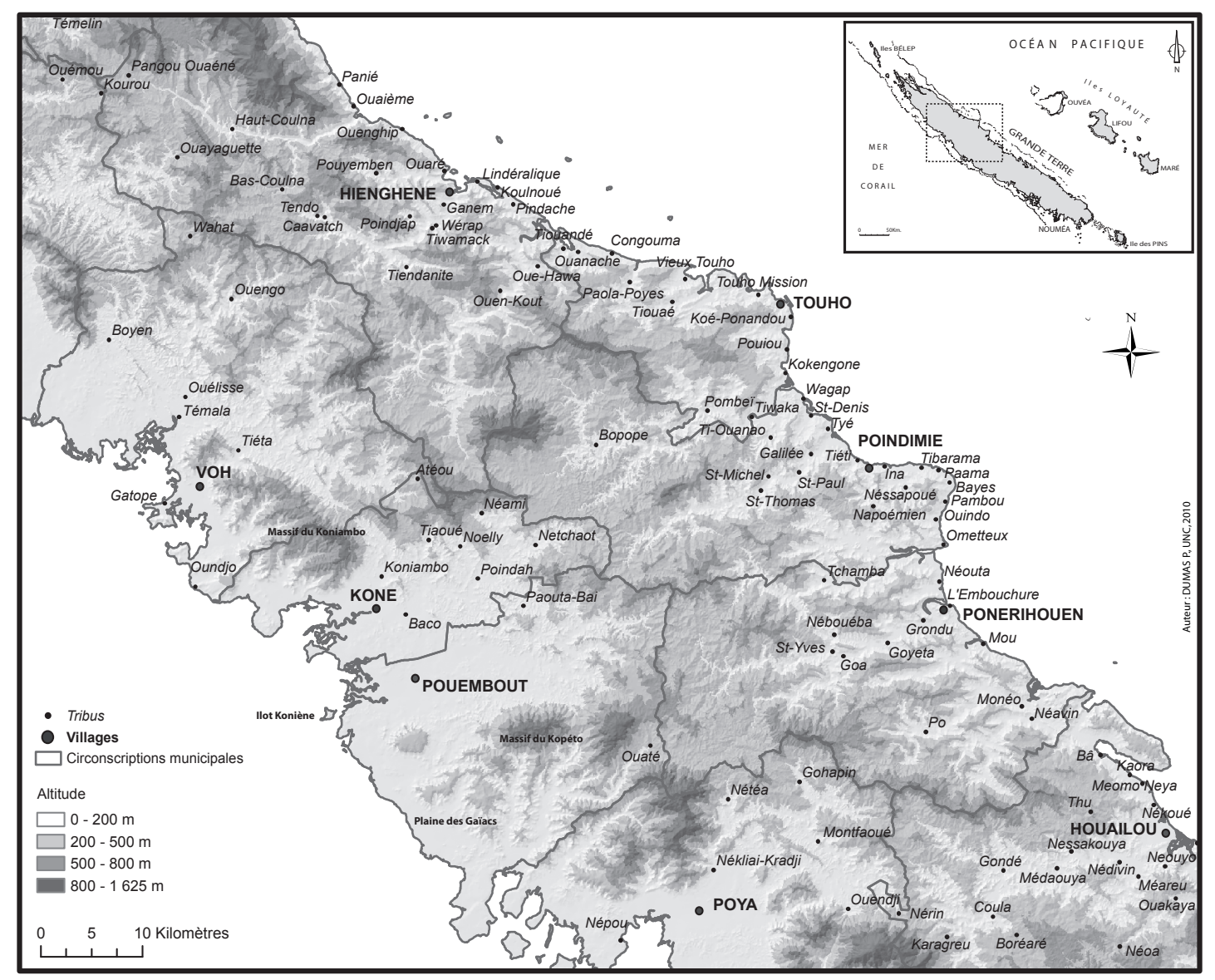

Carte 1. - Le centre-nord de la Grande Terre (C Pascal Dumas, Université de la Nouvelle-Calédonie, 2010)

dans certaines tribus et rejeté dans d'autres ? Les réponses que j'ai collectées à ce sujet renvoient à deux registres particuliers de légitimation, l'un « coutumier», l'autre religieux.

\section{Une histoire de clans}

Netchaot, Néami et Bopope sont, aux côtés d'Atéou, les tribus les plus montagneuses et les plus enclavées de la commune de Koné, situées sur les hauts contreforts (voire, dans le cas de Bopope, à l'intérieur même) de la Chaîne centrale qui sépare les versant ouest et est de la Grande Terre sur toute sa longueur, très loin donc du village européen de Koné qui se trouve à proximité du littoral. À propos de ses premiers passages dans ces trois tribus au nom du Groupe 1878, Élie Poigoune indique que son action et sa personne étaient perçues d'une façon très spécifique par leurs habitants, à l'aune de l'histoire politique antérieure de la région. Se dessine ici un phénomène particulier de remobilisation, dans un contexte contemporain, d'anciennes alliances politiques courant le long d'itinéraires situés au cœur de la Chaîne centrale, des hautes vallées du versant est de la Grande Terre - en particulier depuis la tribu de Poyes (Paola-Poyes sur la carte ci-jointe) d'où Élie Poigoune était originaire, située dans la commune de Touho à celles du versant ouest (Netchaot, Néami) en passant par la tribu intermédiaire de Bopope. Il est particulièrement intéressant de noter que, selon le fondateur du Groupe 1878, lui-même n'avait pas mobilisé volontairement ces liens mais que ce sont les habitants des tribus concernées qui insistèrent auprès de lui sur les raisons « coutumières » de son accueil :

«Les liens coutumiers, ça a beaucoup aidé. À Netchaot, mais même à Bopope. Les gens, ils sont marqués, ils gardent toujours cette image-là. [...] On a des liens coutumiers avec Netchaot. Enfin moi, j’ai redécouvert ça, je les ai pas utilisés, mais quand je me suis présenté, les gens nous ont reçus à bras ouverts et nous ont expliqué les liens qu'on avait avec eux. Jusqu’à Baco, hein, autour. Il y a des familles qui nous ont accueillis parce qu'on était un tel. »

Les travaux d'Alban Bensa sur l'histoire politique des hautes vallées du centre-nord de la Grande Terre permettent de comprendre la configuration précise de ces "liens coutumiers " mobilisés dans le cadre de l'implantation du 


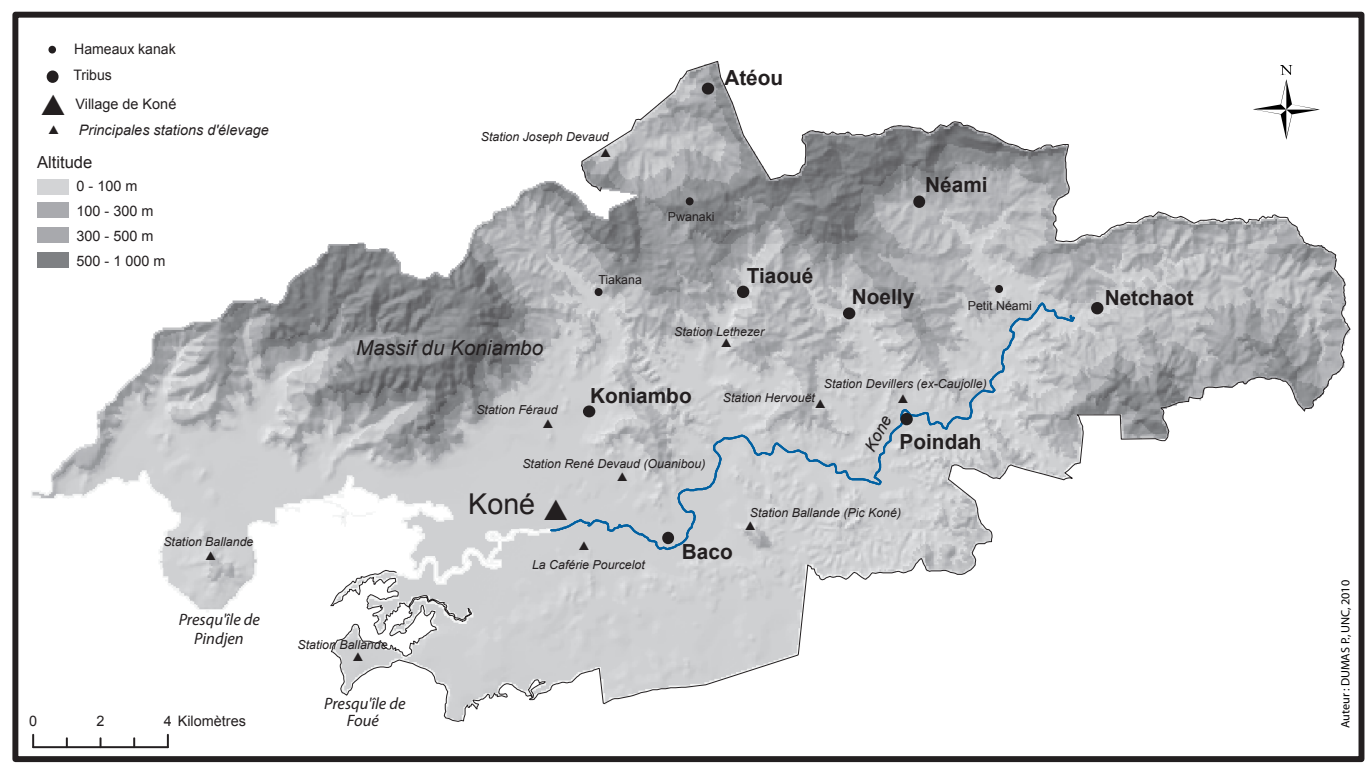

Carte 2. - La circonscription communale de Koné, années 1970 (@ Pascal Dumas, Université de la Nouvelle-Calédonie, 2010)

Palika. Il mentionne notamment deux « clans $»^{13}$ implantés dans les tribus montagneuses de Koné et de Touho, dont les trajectoires historiques croisèrent à plusieurs reprises celles du clan et de la tribu d'Élie Poigoune: d'une part le clan Baraotâ, aujourd'hui concentré, dans la circonscription de Koné, au sein des tribus de Netchaot et de Baco (Bensa et Goromido, 2005 : 50-55; Bensa et al., 2015 : 481-500); d'autre part le clan Garaatü, présent à Koné dans les tribus de Néami, Bopope, Atéou et Baco (Bensa et Rivierre, $1982: 201-220 ; 1994: 31-38)^{14}$. Dans la région de Koné, mis à part le cas particulier de la tribu de Baco située près du bord de mer (Guiart, 1992: 123-136), ces deux groupes étaient historiquement localisés dans les zones centrales de la chaîne montagneuse (plateau de Tango et vallée de la Wéaga). Ils se trouvaient en amont du territoire contrốlé par les clans Görötû et Nädù depuis la fin du XVIII ${ }^{\mathrm{e}}$ siècle dans la moyenne vallée de la Koné, institution politique que l'administration coloniale allait identifier et requalifier en 1870 comme la "grande chefferie de Poindah" (Bensa et Goromido, 2005 : 67$75)^{15}$. Au cours du XIx ${ }^{e}$ siècle (avant et après l'arrivée des Européens), alors qu'ils se situaient politiquement et géographiquement entre les deux ensembles forts que représentaient les chefferies de Poindah au sud-ouest et de Poyes au nord-est, les Garaatü et les Baraotâ firent face à plusieurs événements violents du côté de Koné qui les poussèrent à se réfugier à Poyes.

Ainsi, selon un récit délivré à Netchaot par Antoine Goromido en 1985, des membres du clan Görötû organisèrent un guet-apens à Pwaara (versant est de la Chaîne centrale), probablement dans la première moitié du XIX ${ }^{\mathrm{e}}$ siècle, contre des Garaatü qu'ils avaient invités et qu' ils massacrèrent (Bensa et Goromido, 2005 : 28). Un autre récit recueilli à Poyes dans les années 1960 indique que les Garaatü de la vallée de la Wéaga firent face au milieu du XIX ${ }^{e}$ siècle à la vindicte de la chefferie des Nädù et des Görötû (en la personne de son fameux guerrier Goodu, tué en 1869), et qu'ils durent se réfugier à Poyes (Bensa et Rivierre, 1982 : 133). Enfin, parmi les Baraotâ localisés à cette époque sur le plateau de Tango, selon un récit narré par Siméon Goromido en 1975 à Netchaot, certains lignages firent appel à Goodu pour guerroyer contre d'autres lignages rivaux du mềme clan. Celui-ci combattit finalement l'ensemble des protagonistes: datée de 1865, cette grande bataille de Pwänaacè se solda par la dispersion de nombreux Baraotâ, dont une bonne partie se réfugia vers le nord-est, à Bopope et à Poyes (Bensa et Goromido, 2005 : 52-55).

13. Dans l'organisation segmentaire kanak, un «clan " (terme issu du français local) est un rassemblement de plusieurs groupes lignagers se reconnaissant un ancêtre commun.

14. Ces deux clans sont nommés Baraotâ et Garaatü en paicî (langue parlée dans toutes les tribus de Koné sauf Bopope et Baco) et Bèlèot et Galaahî en cèmuhî (langue parlée à Netchaot, Bopope, ainsi qu’à Poyes et dans les autres tribus de Touho). Les habitants de Netchaot sont à la fois locuteurs du paicî et du cèmuhî. Ceux de Baco parlent le haeke (langue apparentée à celles du bord de mer de Voh).

15. L'armée intervint pour la première fois dans la région de Koné en 1865, après l'attaque de deux navires européens. En 1870, au terme d'une campagne militaire agitée, elle décerna à ses deux alliés principaux les titres de " grands chefs » de Poindah et de Baco. 
Il faut insister sur la temporalité de ces différents récits : Siméon Goromido raconta en détail la bataille de Pwänaacè en 1975, soit cent-dix ans après les événements et quelques mois après la première tournée d'Élie Poigoune à Netchaot. De même, les conflits entre les Nädù-Görötû et les Garaatü furent précisément évoqués dans les années 1960 à Poyes, puis vingt ans plus tard à Netchaot. La bataille de Pwänaacè a en outre fait l'objet de diverses réappropriations mémorielles plus récentes de la part des habitants de Netchaot : poésie publiée en 1992, pièce de théâtre jouée en 1993, stèle dressée à proximité du site (Bensa et Goromido, 2005 : 54-55). On trouve donc ici tous les indices du maintien, jusqu'à la période contemporaine, d'une mémoire locale précise sur quelques grands événements politiques du $\mathrm{XIX}^{\mathrm{e}}$ siècle. L'histoire longue et mouvementée des migrations successives des Baraotâ et des Garaatü de Koné à Poyes fit clairement l'objet d'une transmission de génération en génération au sein des groupes directement concernés par ces événements. Ces éléments soulignent combien, à l'intérieur des tribus montagneuses de Koné, les références politiques anciennes étaient toujours prégnantes lorsque Élie Poigoune mena les premières tournées du Groupe 1878 en 1974. Cette mémoire locale des replis successifs à Poyes fut remobilisée d'autant plus fortement lors des passages d'Élie Poigoune à Netchaot, Bopope et Néami dans les années 1970, que la famille Poigoune bénéficiait d'une position stratégique importante au sein du clan titulaire de la chefferie de Poyes, probablement depuis la fin $\mathrm{XVIII}^{\mathrm{e}}$ siècle; or les lignages accueillis à Poyes au siècle suivant, après les attaques des Nädù-Görötû, nouèrent avec les piliers de cette chefferie des relations matrimoniales privilégiées (Bensa et Rivierre, 1982 : 121, 202-210). Au fil du temps, les cérémonies de mariage et de deuil permirent de manifester et de réaffirmer régulièrement le sens de ces alliances politicomatrimoniales. D'une certaine façon, c'est donc en tant que représentant d'une chefferie ayant historiquement servi de refuge aux leurs à plusieurs reprises, qu'Élie Poigoune fut en retour « reçu à bras ouverts » par les Baraotâ et les Garaatü de Netchaot, Néami et Bopope.

Le fondateur du Groupe 1878 explique aussi l'adhésion d'une partie importante de la population de Néami à son discours politique en évoquant un épisode historique plus récent - la répression de la guerre de 1917 - au cours duquel certains habitants des hauts de Koné vinrent de nouveau se réfugier à Poyes. Les archives européennes sur la guerre de 1917 vont dans le même sens: Poyes constituait effectivement l'une des principales tribus ayant protégé les populations «rebelles " de Néami et Netchaot (Muckle, 2012 : 130-137). Près de soixante ans plus tard, le souvenir de ces migrations était encore intact lorsque Élie Poigoune organisa sa propagande politique dans la région :

"À Néami, il y a beaucoup de gens qui ont vécu à Poyes, pendant la révolte de 1917. [...] Ils ont utilisé les liens, ils sont allés chercher des gens pour fuir la répression. Une fois que c'était fini, les gens sont repartis. Et c'est comme ça qu'on est très..., que les gens nous ont reçus. Parce qu'ils se sont rappelés de cette époque-là, qu'ils ont été accueillis là-haut. Nous on était pas là, mais les vieux nous racontent. "

Il paraît important d'insister ici sur le caractère fondamentalement politique de ces solidarités nouées au XIX ${ }^{e}$ et au début du $\mathrm{XX}^{\mathrm{e}}$ siècles, dont put ainsi bénéficier Élie Poigoune, des décennies plus tard, dans les tribus montagneuses de Koné. Dans cette perspective, la lecture locale de son travail de mobilisation partisane à travers des logiques d'alliances familiales et matrimoniales anciennes témoignait de la continuité d'une perspective politique kanak déployée sur le temps long, avant, pendant et après la période coloniale, depuis la fondation de la chefferie de Poyes au XVIII ${ }^{\mathrm{e}}$ siècle jusqu'à l'irruption du Groupe 1878. L'analyse des conditions de l'entrée du Palika dans les tribus de Koné permet ici de saisir un régime d'historicité politique spécifique au monde kanak, qui recouvrait mais ne se limitait pas au seul moment colonial, auquel fut articulée une autre temporalité politique liée à la lutte pour la décolonisation et l'indépendance.

\section{Une histoire de religion}

À Koné, l'identification locale d'Élie Poigoune renvoyait également à son statut particulier de fils du pasteur en poste à Noelly. En effet, vers 1964-1965, alors qu'il partait faire ses études en métropole, son père Roch Poigoune fut nommé à la tête de la paroisse protestante de cette tribu. Les tournées d'Élie Poigoune à Koné étaient semblet-il appréhendées localement autant à l'aune de cet héritage familial religieux bien spécifique, qu'en fonction de son appartenance clanique. En 1974, le vécu commun de ses parents et de ses frères avec les habitants de Noelly depuis une dizaine d'années permit par exemple au fondateur du Groupe 1878 de pénétrer facilement dans la tribu pour y organiser une réunion d'information politique. Interrogé en 2004, l'un des premiers militants Palika de Noelly insistait sur sa familiarité vis-à-vis des gens de la tribu :

BT : «Comment ça s’est passé tes débuts en politique, au Palika?"

Moïse Gowécée : «C'est par Élie, il venait à Noelly pour les vacances, on était tout le temps avec lui. Il venait, et puis on discutait. C'est comme ça qu'il a fait la première réunion, ça s'est passé en 74. » (Moïse Gowécée, entretien du 29 septembre 2004, tribu de Noelly, Koné) 
Depuis qu'une crise missionnaire avait divisé le monde protestant calédonien en 1957-1958, les Kanak protestants se rattachaient soit à l'Église évangélique "autonome " (héritière de la Société des missions de Paris), soit à l'Église évangélique "libre" dissidente (Trépied, 2010: 166-170). La commune de Koné comptait quatre tribus protestantes "autonomes" (Baco, Bopope, Netchaot et Noelly), quatre tribus catholiques (Atéou, Koniambo, Néami et Tiaoué) et une tribu protestante « libre » (Poindah) ${ }^{16}$. Les réseaux locaux de l'Église évangélique autonome se révélèrent cruciaux dans l'élaboration des itinéraires empruntés à Koné par le Groupe 1878 en 19741975 : les habitants de Noelly retrouvaient en effet fréquemment ceux de Netchaot, Bopope et Baco lors des nombreuses activités religieuses organisées à l'échelle du consistoire. Les Kanak des tribus protestantes autonomes de Koné connaissaient donc personnellement les membres de la famille Poigoune installés à Noelly. En 2002, l'un des premiers militants Palika de Bopope évoquait ces liens en décrivant la préparation de la première réunion du Groupe 1878 dans sa tribu :

"C'est Daniel, tout ça, c'est la bande, les frères à Élie. Ils sont venus ici par rapport à pasteur, parce que leur père, Roch Poigoune, était pasteur. Ça fait que nous on se connaît comme ça, à cause de leur père. [...] Ils connaissent les gens d'ici parce qu'ils sont toujours ensemble quand il y a les fêtes de l'Église. " (Philippe Poaracagu, entretien du 25 novembre 2002, tribu de Bopope, Koné)

Points d'ancrage stratégiques à Noelly, Baco, Netchaot et Bopope, les relations entre protestants autonomes pouvaient ainsi être mobilisées en articulation avec les liens claniques dont bénéficiait simultanément le fondateur du Groupe 1878. Localement, la mobilisation combinée de ces deux types de réseaux sociaux ouvrait certaines portes à Élie Poigoune et lui en fermait d'autres. À Baco, Bopope et Netchaot, il pouvait facilement emprunter un double chemin coutumier et religieux grâce aux Baraotâ et Garaatü protestants auxquels il était étroitement lié. À Noelly, les liens religieux noués au quotidien entre sa famille et les habitants de la tribu permettaient de compenser la relative faiblesse de ses appuis claniques dans ce bastion politique de la grande chefferie de Poindah. En revanche, ces réseaux n'étaient d'aucune utilité dans les tribus catholiques de Koniambo et Tiaoué, dont les habitants étaient pour la plupart inscrits dans d'autres systèmes d'alliance, tandis qu’à Atéou et Néami, la présence d'alliés coutumiers pouvait permettre d'amoindrir le cloisonnement découlant des appartenances religieuses divergentes. Enfin Élie Poigoune ne disposait d'aucune "clé " religieuse pour pénétrer dans la tribu protestante libre de Poindah, par ailleurs siège d'une grande chefferie géographiquement et politiquement éloignée de celle de Poyes.

$\grave{A}$ ce stade de l'analyse, les articulations complexes entre liens coutumiers, religieux et militants à l'œuvre lors du lancement du Palika à Koné, offrent donc une compréhension renouvelée des logiques politiques kanak. En adoptant une perspective de longue durée sur l'histoire régionale, on peut en effet retracer toute une série de connections et de réactions en chaîne entre plusieurs événements à première vue distincts, qui met au jour un véritable univers kanak spécifique du politique, évoquant des "répertoires vernaculaires du politique " (Bertrand, 2008) particulièrement opérants. Les récits sur les conflits précoloniaux, les modalités pratiques de l'évangélisation, les processus d'implantation des chefferies administratives et les logiques de fragmentation partisane dans les années 1970, lorsqu'ils sont pensés ensemble, dessinent des configurations locales de rapports de force qui se redéfinissent, se reproduisent et se transforment, au gré des contextes historiques et des ressources sociales mobilisables dans une situation donnée. Ainsi, plusieurs décennies avant que la propagande du Groupe 1878 ne circule à Koné le long des chemins coutumiers ou religieux, la pénétration de la parole protestante dans la zone au début du siècle avait elle-même répondu à des rationalités politiques similaires renvoyant aux alliances entre les Baraotâ, les Garaatü et la chefferie de Poyes. L'évocation par Élie Poigoune du protestantisme dans la région de Touho-Koné laisse apparaître certaines des logiques politiques et sociales sur lesquelles reposa ultérieurement son action militante :

«La religion, c'est arrivé un peu comme ça. À Touho, il y avait deux chefs qui faisaient la guerre, dans les années 1900 . Il y en avait un catholique, et l'autre chef à nous, à Poyes, il était encore dans la forêt, il avait pas pris la religion. [...] Ils ont été jugés par Feillet, le gouverneur, à Pamalé [dans la Chaîne centrale] [...] Il leur a dit: "toi, le chef de Touho, tu es déjà catholique. Toi, le chef qui vis dans la forêt, ta punition, c'est être protestant." Quand il lui a dit d'être protestant, il est descendu... Parce que la chefferie de Poyes, ça allait jusqu'à Netchaot, Bopope, Pombéi, tout ça, tout le bord de mer. Il a dit à ses gens, les vieux, "vous allez partir voir la religion", la religion protestante était déjà à Tibarama [tribu côtière située au sud-est, à Poindimié] [...]. Ils sont partis les vieux, ils ont marché quelques jours, ils sont arrivés à Tibarama, ils ont fait la coutume. Les autres là-bas, ils ont fait : "voilà la religion", ils ont donné une Bible, avec un bout de manou [tissu échangé lors des cérémonies]. Ils sont revenus, et ils ont donné au chef. Le bout de tissu, il l'a coupé en plusieurs morceaux. Il y a un morceau qui est tombé à Netchaot, un qui est 
tombé à Pombéi, un qui est arrivé à Bopope, un qui est arrivé sur tout Touho, les tribus, il a distribué. Et à partir de ce moment-là, on est devenu protestants. Et donc c'est comme ça, à Netchaot ils sont protestants, c'est comme ça que c'est arrivé. Donc ils ont pris la religion, et ils ont dit: "ben voilà, nous on a une Bible, on va envoyer un jeune, pour aller apprendre ce que c'est." Et ils ont envoyé mon père, tout jeune. Ils lui ont dit : "tu vas aller à Do Néva" [mission protestante] [...], tu vas apprendre la religion." Lui, il est allé. [...] Et donc j’ai grandi là-bas, je suis né là-bas. Jusqu'à l'âge de 10 ans, je suis resté à Do Néva. "

Si l'on suit ce récit, les conflits entre chefferies rivales de Touho, arbitrés par l'administration, déterminèrent les modalités de la diffusion du protestantisme à travers la Chaîne centrale. Ils influèrent simultanément sur le destin historique de la lignée Poigoune, désormais chargée, dans ce contexte régional reconfiguré, d'une nouvelle tâche sociale et politique relative aux activités religieuses protestantes. Les ressources sociales attachées à cette fonction se transmirent et se transformèrent à la génération suivante sous la forme de dispositions inédites contribuant à un phénomène d'ascension sociale: mobilités géographiques, décloisonnement social, plurilinguisme, enfin et surtout capital scolaire. Concrètement, le pasteur Roch Poigoune, son épouse et ses enfants vécurent successivement à Touho, Houaïlou (Do Néva), Canala et Koné, où ils apprirent à chaque fois les langues locales, tandis que la trajectoire étudiante et professionnelle d'Élie Poigoune incluait en plus Nouméa et la métropole. Le fondateur du Groupe 1878 s'inscrivit finalement dans cet héritage politique en le reconfigurant à l'aune d'outils analytiques et organisationnels nouveaux et d'objectifs stratégiques inédits.

D’une certaine façon, les débuts du Palika dans les tribus des hauts de Koné au milieu des années 1970 signifia donc la prise en charge réussie de ce système politique kanak par Élie Poigoune. Indépendamment de son discours militant, voire de sa propre volonté, il était d'abord perçu à Koné comme l'héritier de multiples dynamiques locales de pouvoir, dans le cadre d'une histoire politique régionale indissociablement clanique, religieuse et partisane. Larticulation de ces diverses rationalités et temporalités dessinaient des chemins d'alliance spécifiques dans la région, qui déterminaient largement la cartographie locale du Palika.

\section{Conclusion}

Dans les années 1970, la stratégie politique développée par Élie Poigoune et les dirigeants du Palika représentait un cas exceptionnel, dans l'histoire calédonienne, de constitution raisonnée d'un parti politique n'émanant pas d'autres structures collectives - en particulier des structures religieuses, comme cela avait été le cas pour l'uc après la guerre. Ce projet militant, volontairement pensé et construit par les premiers diplômés kanak en dehors des contingences sociales locales, reposait notamment sur une stratégie méthodique de mobilisation auprès des "jeunes ", lycéens et étudiants, travailleurs urbains et habitants des tribus. Pourtant, malgré cette genèse intellectuelle unique, le lancement du Palika à Koné au milieu des années 1970 ne s'est pas déroulé sur un terrain vierge, mais dans un espace social maillé par un ensemble de relations coutumières, religieuses et familiales pré-existantes, renvoyant à des régimes d'historicité distincts, qui a entièrement saisi la nouveauté partisane. C'est au croisement de multiples clivages entre générations et entre tribus, nés des nombreux échos du passé diversement appréhendés par les Kanak de Koné dans les années 1970, que se construisaient concrètement les logiques d'implantation du nouveau parti. Qu'ils le veuillent ou non, les leaders étudiants ont dû se conformer à cette appréhension kanak locale du politique irriguant tous les secteurs de la vie sociale - y compris leurs propres trajectoires - au-delà de la seule rhétorique militante et idéologique.

Ces éléments de réflexion permettent de comprendre globalement la structuration du parti à l'échelle de la région de Koné-Touho, mais ils n'éclairent au final qu'un aspect de la complexité du "phénomène Palika ». En resserrant la focale à l'échelle d'une tribu, d'une maisonnée, d'une famille ou d'un individu, l'opposition uc/Palika révèle en effet de nouvelles logiques sociales de différenciation, dont je ne peux rendre compte ici faute de place (voir cependant Trépied, 2007 : 782-821). Au-delà de Koné, le lancement du parti dans d'autres régions mobilisait également des liens sociaux particuliers, qu' il semble difficile d'observer à une autre échelle que locale. Si la question des mécanismes sociaux au fondement de la distinction uc/Palika s'avère donc pertinente à quelque échelle d'analyse que ce soit, elle semble néanmoins appeler des réponses très complexifiées, au vu de la forte fragmentation sociale et politique du monde kanak. Dans ces conditions, une histoire sociale générale du Palika pourrait être produite à travers la compilation de plusieurs ethnographies historiques localisées du Palika, à Koné et dans d'autres contextes locaux, associée à l'analyse du travail d'homogénéisation politique de ces diversités sociales locales réalisé par l'appareil militant central.

\section{BIBLIOGRAPHIE}

Bensa Alban et Antoine Goromido, 2005. Histoire d'une chefferie kanak. Le pays de Koohnê (Nouvelle-Calédonie), Paris, Karthala. 
Bensa Alban, Kacué Yvon Goromoedo et Adrian Muckle, 2015. Les sanglots de l'aigle pêcheur. Nouvelle-Calédonie : la guerre kanak de 1917, Toulouse, Anacharsis.

Bensa Alban et Jean-Claude Rivierre, 1982. Les chemins de l'alliance. L'organisation sociale et ses représentations en Nouvelle-Calédonie, Paris, SELAF.

-, 1994. Les filles du Rocher Até. Contes et récits paicî, Paris, Geuthner-ADCK.

BERTRAND Romain, 2006. Les sciences sociales et le "moment colonial»: de la problématique de la domination coloniale à celle de l'hégémonie impériale, Questions de Recherche 18, CERI-Sciences Po, pp. 1-41.

—, 2008. Politiques du moment colonial. Historicités indigènes et rapports vernaculaires au politique en "situation coloniale", Questions de Recherche 26, CERI-Sciences Po, pp. 1-49.

Chappell David, 2003. The Kanak Awakening of 1969-1976: Radicalizing Anti-Colonialism in New Caledonia, Journal de la Société des Océanistes 117 : 150 ans de colonisation en Nouvelle-Calédonie (I. Leblic éd.), pp. 187202 (https://jso.revues.org/1268).

-,2013, The Kanak Awakening. The Rise of Nationalism in New Caledonia, Honolulu, University of Hawai i Press, Pacific Islands Monograph Series 27.

Dauphiné Joël, 1989. Les spoliations foncières en Nouvelle-Calédonie (1953-1913), Paris, L'Harmattan.

Demmer Christine, 2002. Les héritiers d'Éloi Machoro (1941-1985). Une génération nationaliste au pouvoir à Ammââ et Kûöö, villages de Xârâcùù (Canala), Nouvelle-Calédonie, Paris, EHess, thèse de doctorat en anthropologie.

—, 2003. Nationalisme kanak et génération : une approche du changement social en Nouvelle-Calédonie, colloque Les approches générationnelles: enjeux, avancées, débats, Université Versailles-Saint-Quentin, (http://www. recherches-nouvelle-caledonie.org/IMG/ pdf/Nationalisme_kanak_et_generation.pdf, consulté le 23 décembre 2016).

-, 2016. Socialisme kanak. Une expérience politique à Canala (Nouvelle-Calédonie), Paris, Karthala, Karapaa.

Freyss Jean, 1995. Économie assistée et changement social en Nouvelle-Calédonie, Paris, PUF, Tiers-Monde/IEDEs.

Guiart Jean, 1992 (2e éd. remaniée et augmentée; $1^{\mathrm{e}}$ éd. 1963). Structure de la chefferie en
Mélanésie du Sud, Paris, Institut d'ethnologie, musée de l'Homme.

Guiart René, 2001. Le feu sous la marmite, Nouméa, Le Rocher à la Voile.

Journal de la Société des Océanistes, 1995. Mélanésia 2000 - Dossiers, documents et témoignages, Journal de la Société des Océanistes 100101 (http://jso.revues.org/persee-246564).

Kurtovitch Ismet, 1997. Aux origines du FLNKS, l'UICALO et l'AICLF (1946-1953), Nouméa, île de Lumière.

Leblic Isabelle, 1993. Les Kanak face au développement. La voie étroite, Grenoble, ADCKPresses universitaires de Grenoble.

—, 2003. De la démocratie à la base : coutume et militantisme Kanak dans les années 19851986, suivi de la généalogie du paysage politique en Nouvelle-Calédonie (1946-1988), in Jean-Marc Regnault (éd.), François Mitterrand et les territoires français du Pacifique (19811988). Mutations, drames et recompositions; enjeux internationaux et franco-français, Les Indes savantes, Paris, pp. 311-317 et pp. 318-319.

Mannheim Karl, 1990 [1928]. Le problème des générations, Paris, Nathan, Essais et recherches.

Merle Isabelle, 2004. De la « légalisation" de la violence en contexte colonial. Le régime de l'indigénat en question, Politix 66, pp. 137-162.

MucKLE Adrian, 2012. Specters of Violence in a Colonial Context: New Caledonia, 1917, Honolulu, University of Hawai'i Press.

Naepels Michel, 1998. Histoires de terres kanakes. Conflits fonciers et rapports sociaux dans la région de Honaïlou (Nouvelle-Calédonie), Paris, Belin, Socio-histoires.

NéAoutyine Paul, 2006. L'Indépendance au présent. Identité kanak et destin commun, Paris, Syllepse.

Revel Jacques (éd.), 1996. Jeux d'échelles. La micro-analyse à l'expérience, Paris, Hautes Études, Gallimard, Le Seuil.

SALAÜN Marie, 2009. Une parentalité disqualifiée? Être jeunes parents kanak en Nouvelle-Calédonie aujourd'hui, in $\mathrm{N}$. Gagné et L. Jérôme (éds), Jeunesses autochtones. Affirmation, innovation et résistance dans les mondes contemporains, Rennes, Presses universitaires de Rennes, pp. 79-96.

Saussol Alain, 1979. L'Héritage. Essai sur le problème foncier mélanésien, Paris, Société des Océanistes, Publications de la Société des 
Océanistes 49 (http://books.openedition.org/ sdo/563).

Soriano Éric, 2001. Une trajectoire du politique en Mélanésie. Construction identitaire et formation d'un personnel politique. L'exemple kanak de Nouvelle-Calédonie (1946-1999), Montpellier, Université Montpellier 1, thèse de doctorat en science politique.

-, 2014, La fin des Indigènes en Nouvelle-Calédonie. Le colonial à l'épreuve du politique, 1946-1976, Paris, Karthala, Karapaa.
Tuibaou Jean-Marie, 1996. La présence kanak, Paris, Odile Jacob.

TrépIed Benoît, 2007. Politique et relations coloniales en Nouvelle-Calédonie. Ethnographie historique de la commune de Koné, 1946-1988, Paris, EHess, thèse de doctorat en anthropologie.

-, 2010. Une mairie dans la France coloniale. Koné, Nouvelle-Calédonie, Paris, Karthala, Recherches internationales-province Nord. 